\title{
Neural Flip-Flops I: Short-Term Memory
}

2 Lane Yoder

3 Department of Science and Mathematics, retired

4 University of Hawaii, Kapiolani

5 Honolulu, Hawaii

6 LYoder@hawaii.edu

$7 \quad$ NeuralNanoNetworks.com 
8

9 The networks proposed here show how neurons can be connected to form flip-flops, the basic

10 building blocks in sequential logic systems. Two novel neural flip-flops (NFFs) are composed of

11 two and four neurons. Their operation depends only on minimal neuron capabilities of excitation

12 and inhibition. The NFFs can generate known phenomena of short-term memory. Memory tests

13 have shown that certain neurons fire continuously at a high frequency while information is held

14 in short-term memory. These neurons exhibit seven characteristics associated with memory

15 formation, retention, retrieval, termination, and errors. One of the neurons in each of the NFFs

16 produces all of the characteristics. This neuron and a second neighboring neuron together

17 predict eight unknown phenomena. These predictions can be tested by the same methods that led

18 to the discovery of the first seven phenomena.

19 Key words: flip-flop; short-term memory; neuronal network; neural network; neural correlate;

20 working memory; neural logic circuit; explicit neural model; color vision; olfaction. 


\section{Introduction}

This article is the fourth in a series of articles that show how neurons can be connected to process information. The first three articles [1-3] explored the analog properties of neuron signals in combinational logic operations, whose outputs depend only on the current state of the inputs. A fuzzy logic decoder was shown to generate the major phenomena of both olfaction and color vision (such as color mixing and the shape of color space), including the brain's shortcomings (such as the Bezold-Brücke hue shift) [1,2]. The decoder's design is radically different from a standard electronic digital (Boolean logic) decoder $[2,4,5]$. If implemented with electronic components and given digital inputs, the decoder performs the same Boolean function as the standard digital design more efficiently.

It was shown that a single neuron with one excitatory input and one inhibitory input, with signal strengths $\mathrm{X}$ and $\mathrm{Y}$, respectively, can function as a logic primitive, $\mathrm{X}$ AND NOT Y [2]. In simplest terms, this is because the neuron is active when it has excitatory input and does not have inhibitory input. It was also shown that an AND-NOT gate can be configured to function as an inverter (i.e., a NOT X logic primitive). The AND-NOT gate together with a NOT gate make up a functionally complete set, meaning any logic function can be performed by a network of such components. The neural AND-NOT gate will be reviewed and used in the networks proposed here.

The present article considers the Boolean logic properties of neuron signals in sequential logic operations, whose outputs are functions of both the current inputs and the past sequence of inputs. That a neuron can operate as a functionally complete logic gate, analog or digital, provides a framework for the brain's processing of information - analog and digital, combinational and sequential.

Flip-flops are the basic building blocks of sequential logic systems. A flip-flop is a mechanism that can be set repeatedly to either one of two stable states, commonly labeled 0 and 
1. A flip-flop can be used as a memory mechanism to store one bit of information. It is shown here that a few AND-NOT gates can be connected to perform the same function as two standard electronic flip-flops, an active low and active high Set-Reset (SR) flip-flop. These are not the only flip-flops that can be constructed with AND-NOT gates, but they may be the simplest. The network designs are modifications of standard electronic logic circuit designs. The modifications are necessary to implement the circuits with neurons because the AND-NOT gate is virtually never used as a building block in electronic computational systems.

The NFFs produce known and unknown phenomena of short-term memory. With inputs from the outputs of NFFs, neural decoders proposed in [2] can retrieve encoded information that is held in NFFs. That is, a memory can be recalled. The NFFs' robust operation is demonstrated by simulation, but the properties can be proven directly from the explicit network connections and minimal neuron properties of excitation and inhibition.

The NFFs' operation is dynamic, meaning the only changes are the levels of neuron activity. No structural change is required, such as neurogenesis, synaptogenesis, or pruning, nor is any change required in the way neurons function, such as a change in synaptic strength or the strength of action potentials. This makes the networks' speed consistent with the "real time" of most brain functions (a few milliseconds). The NFFs' architectures are explicit, meaning all neurons, connections, and types of synapses are shown explicitly, and all assumptions of neuron capabilities are stated explicitly. Only minimal neuron capabilities are assumed, and no network capabilities are assumed.

\section{Unexplained phenomena and previous models}

There is no consensus on a single neuron's basic logic capabilities or on the brain's organization of synaptic connections at the local level. Consequently, many brain phenomena lack explicit explanations. 


\subsection{Single neuron logic capability}

McCulloch and Pitts' seminal paper [6] proposed that the brain is made up of logic gates. The idea of Boolean neurons had a tremendous effect on artificial neural networks and machine learning, but it had a limited impact on neuroscience [7]. More than 70 years later, the brain's computational capabilities are still unclear [8]. In that time span, many theoretical models have been proposed for neuron responses as mathematical or logic functions, but the modern view of these models follows "the adage that all models are wrong, but some are useful" [9].

The neuron response model used here (and in [1-3]) shows that a neuron with one inhibitory input that can suppress one excitatory input can function as a logic primitive that is sufficient for all logic operations. Apparently there is no other claim in the literature that a single neuron can function as a specific logic primitive based on the minimal neuron capabilities of excitation and inhibition.

\subsection{Short-term memory}

\subsubsection{Known memory phenomena}

Memory tests have shown that certain neurons fire continuously while information is held in short-term memory. This activity was found in neurons in the visual, auditory, and sensorimotor cortexes of monkeys while corresponding sensory information is held in memory $[10,11]$. Similar activity has been found more recently in humans [12].

In the first experiments $[10,11]$, seven characteristics of neural activity were associated with memory formation, retention, retrieval, termination, and errors: 1) Before the stimulus was presented, the sampled neuron discharged at a low, baseline level. 2) When the stimulus was presented, or shortly after, the neuron began to fire at a high frequency. 3) The high frequency firing continued after the stimulus was removed. 4) The response was still high when the memory was demonstrated to be correct. 5) The response returned to the background level 
shortly after the test. 6) In the trials where the subject failed the memory test, the high level firing had stopped or 7) had never begun.

Several models have been proposed for memory mechanisms composed of neurons [1315]. Considerable progress has been achieved, notably with models based on synaptic strength changes [16-19]. However, apparently none of these models is dynamic and explicit, and apparently none produces the seven phenomena of short-term memory described above. The memory bank of NFFs presented here produces all of the phenomena.

\subsubsection{Testable predictions of unknown phenomena}

This article ends with several testable predictions that are implied by the models, briefly outlined here. Since the proposed networks are explicit, any of them can be constructed with actual neurons and tested for specific predicted behaviors. As noted above, one of an NFF's two outputs produces all seven characteristics of neuron activity while information is held in shortterm memory. NFFs predict eight additional phenomena for the pair of outputs. These predictions can be tested by the same methods that led to the discovery of the first seven phenomena. The two NFF output neurons are predicted to have 1) close proximity; 2) reciprocal, 3) inhibitory inputs; 4) complementary outputs; and 5) noise-reducing responses to the inputs. When the memory state is changed, 6) the neuron with high output changes first with 7) the other changing a few milliseconds later. 8) After the memory test, the outputs of both neurons are low.

\section{Simulation methods}

A single-transistor AND-NOT gate was simulated in CircuitLab. The graph of its response function and related plane were created in MS Excel and MS Paint. An NFF was simulated in MS Excel. For this simulation, the number $t_{i}$ represents the time after i neuron 
117

118

119

120

delay times. The neurons' outputs are initialized in a stable state at time $t_{0}=0$. For $i>0$, the output of each neuron at time $t_{i}$ is computed as a function of the inputs at time $t_{i-1}$.

\section{Analysis}

\subsection{Figure symbols}

For several reasons, the neural networks in the figures are illustrated with standard (ANSI/IEEE) logic symbols rather than symbols commonly used in neuroscience schematic diagrams. A comparison is shown in Fig 1.

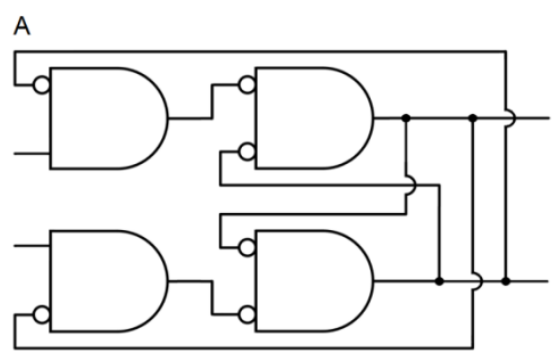

B

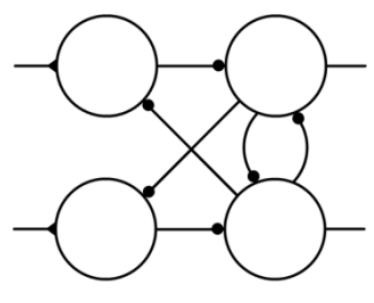

Fig 1. Network symbols. A. A logic circuit illustrated with standard logic symbols. Each of the four components represents a logic function that can be implemented with electronic hardware or with a single neuron. B. The same logic circuit illustrated with symbols commonly used in neuroscience schematic diagrams.

The symbols in Fig 1A can be interpreted in two ways. As a logic symbol, the rectangle with one rounded side represents the AND logic function, and a circle represents negation. So the networks in the figures can be constructed with ordinary electronic components or simulated with electronic circuit software. Second, it will be shown that the logic gate represented by an AND symbol and a circle can be implemented by a single neuron, with a circle representing inhibitory input and no circle representing excitatory input. As shown in Fig 1B, neurons are often represented by a circle, inhibition by a small closed circle, and excitation by a closed triangle, but there does not seem to be an accepted standard of symbols for networks of neurons. 

computational systems means digital signal processing. Neurons can convey analog signals, either with signals of graded strength or with the strength of signals consisting of spikes measured by spike frequency. It will be shown that the neural networks in the figures can

142 generate robust digital signals, i.e., signals with only high and low strengths (except during transition from one state to the other). implemented with neurons, and diagrams of standard logic circuits for the same functions implemented electronically, are easier to see if they are both illustrated with the same symbols. axons than the multiple output channels of Fig 1B. connection, the logic function of each component, the distinction between feedback (right to left) and feed-forward (left to right) signals, and the overall direction of a network's signal processing from input to output (left to right).

\subsection{Neuron signals}

All results for the networks presented here follow from the neuron response to binary 


\subsubsection{Binary neuron signals}

163

164

165

166

167

168

169

170

171

172

173

Neuron signal strength, or intensity, is normalized here by dividing it by the maximum possible intensity for the given level of adaptation. This puts intensities in the interval from 0 to 1, with 0 meaning no signal and 1 meaning the maximum intensity. The normalized number is called the response intensity or simply the response of the neuron. Normalization is only for convenience. Non-normalized signal strengths, with the highest and lowest values labeled Max $\&$ Min rather than 1 and 0 , would do as well.

The responses 1 and 0 are collectively referred to as binary signals and separately as high and low signals. If 1 and 0 stand for the truth values TRUE and FALSE, neurons can process information contained in neural signals by functioning as logic operators. As noted above, the strength of a signal consisting of action potentials, or spikes, can be measured by spike frequency. A high signal consists of a burst of spikes at the maximum spiking rate.

For binary signals, the response of a neuron with one excitatory and one inhibitory input is assumed to be as shown in Table 1 . Of the 16 possible binary functions of two variables, this table represents the only one that is consistent with the customary meanings of "excitation" and "inhibition." 


\begin{tabular}{|c|c|c|}
\hline Excitatory X & Inhibitory Y & Response \\
\hline 0 & 0 & 0 \\
\hline 0 & 1 & 0 \\
\hline 1 & 0 & 1 \\
\hline 1 & 1 & 0 \\
\hline
\end{tabular}

Table 1. Neuron response to binary inputs. The table is also a logic truth table, with the last column representing the truth values of the statement X AND NOT Y.

Some of the components in the figures require continuous, high input. This input is represented by the logic value "TRUE." For an electronic logic circuit, the high input is normally provided by the power supply. If the components represent neurons, the high input can be achieved by neurons in at least four ways. 1) A continuously high signal could be provided by a neuron that has excitatory inputs from many neurons that fire independently [20]. 2) Neurons that are active spontaneously and continuously without excitatory input are known to exist $[21,22]$. A network neuron that requires a high excitatory input could receive it from a spontaneously active neuron, or 3) the neuron itself could be spontaneously active. 4) It will be seen that the high input could be provided by one of a flip-flop's outputs that is continuously high.

\subsubsection{Additive noise in binary neuron signals}

This section covers a potential problem for neural processing of digital (Boolean) information: Additive noise in binary inputs may affect the intended binary outputs of Table 1 . The section includes three main points: Evidence indicates that some neurons have at least some rudimentary noise-reducing capabilities. For the NFF properties obtained here, noise can be sufficiently reduced by neurons that have two simple properties that generalize the noisereducing properties of sigmoid functions. These properties do not indicate capabilities of sophisticated mathematics. 


\subsubsection{Noise reduction}

Two lines of evidence indicate that some neurons have at least a minimal capability of reducing moderate levels of additive noise in binary inputs. The high and low firing frequency associated with memory [10-12] and discussed above is itself evidence of a noise-reducing property. Without some noise-reducing capability, it would be difficult if not impossible for a network to maintain a variable output that can be either high or low. The cumulative effect of additive noise would quickly attenuate the output strength to a random walk through intermediate levels. This is the reason that simple noise-reducing nonlinearities are intentionally built into the materials in electronic components for digital signal processing, as demonstrated below by a single transistor's response.

Second, some neurons are known to have sigmoid responses to single inputs, including inhibitory inputs [23-25]. A sigmoid response reduces moderate levels of additive noise in a binary input signal by producing an output that decreases an input near 0 and increases an input near 1. It will be demonstrated by simulation that a neuron response that is sigmoid in both excitatory and inhibitory inputs is sufficient for the noise-reducing requirements of the NFFs presented here. But such a response is not necessary; a simpler, more general property is sufficient.

Reduction of noise in both excitatory and inhibitory inputs can be achieved by a response function of two variables that generalizes a sigmoid function's features. The noise reduction need only be slight for the proposed NFFs because they have feedback loops that continually reduce the effect of noise.

Let $\mathrm{F}(\mathrm{X}, \mathrm{Y})$ represent a neuron's response to an excitatory input $\mathrm{X}$ and an inhibitory input Y. The function must be bounded by 0 and 1 , the minimum and maximum possible neuron responses, and must satisfy the values in Table 1 for binary inputs. For other points $(\mathrm{X}, \mathrm{Y})$ in the unit square, suppose F satisfies: 
1. $F(X, Y)>X-Y$ for inputs $(X, Y)$ near $(1,0)$ and

2. $F(X, Y)<X-Y$ or $F(X, Y)=0$ for inputs $(X, Y)$ near the other three vertices of the unit square.

The neuron responses of Table 1 are $\max \{0, \mathrm{X}-\mathrm{Y}\}$ (the greater of 0 and $\mathrm{X}-\mathrm{Y}$ ). For binary inputs with moderate levels of additive noise that makes them non-binary, conditions 1 and 2 make the output either closer to, or equal to, the intended output of Table 1 than $\max \{0, \mathrm{X}-\mathrm{Y}\}$. Neurons that make up the networks proposed here are assumed to have these minimal noisereducing properties.

Conditions 1 and 2 are sufficient to suppress moderate levels of additive noise in binary inputs and produce the NFF results found here. The level of noise that can be tolerated by the NFFs depends on the regions in the unit square where conditions 1 and 2 hold. If a binary input $(\mathrm{X}, \mathrm{Y})$ has additive noise that is large enough to change the region in which it lies, an error can occur.

\subsubsection{Example of a neuron response that satisfies conditions 1 and 2}

For any sigmoid function $\mathrm{f}$ from $\mathrm{f}(0)=0$ to $\mathrm{f}(1)=1$, the following function has the noisereducing properties 1 and 2 and also satisfies Table 1:

$$
F(X, Y)=f(X)-f(Y) \text {, bounded below by } 0 \text {. }
$$

This function is plausible as an approximation of a neuron response because it is sigmoid in each variable and some neurons are known to have sigmoid responses to single inputs, as mentioned above. The same sigmoid function applied to $\mathrm{X}$ and $\mathrm{Y}$ is not necessary to satisfy conditions 1 and 2. The function F could be the difference of two different sigmoid functions.

The function $\mathrm{F}$ is illustrated in Fig 2 for a specific sigmoid function $\mathrm{f}$. The sine function of Fig 2A was chosen for $\mathrm{f}$ rather than any of the more common examples of sigmoid functions 
to demonstrate by simulation that a highly nonlinear function is not necessary for robust maintenance of binary signals. On half of the unit square, where $\mathrm{Y} \geq \mathrm{X}$, Fig $2 \mathrm{~B}$ shows that $\mathrm{F}$ has the value 0 . This reflects the property that a large inhibitory input generally suppresses a smaller excitatory input.

A

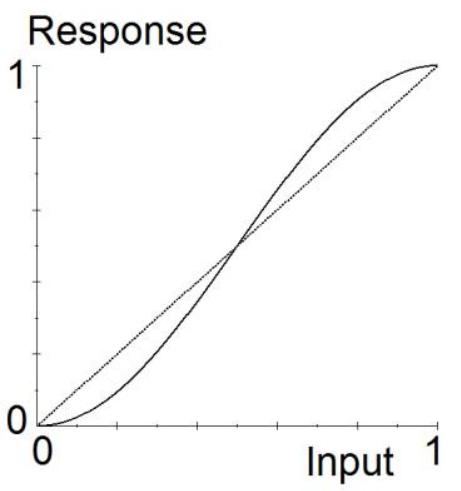

B

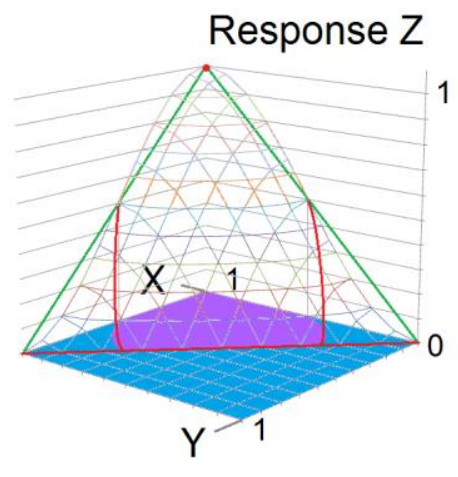

Fig 2. Noise-reducing AND-NOT function. The graphs show an example of a neuron response to analog inputs that reduces moderate levels of additive noise in binary inputs. A. A sigmoid function $\mathrm{f}(\mathrm{x})=(1 / 2) \sin (\pi(\mathrm{x}-1 / 2))+1 / 2$. B. Graph of a function that has the noisereducing properties 1 and 2 . The function is $F(X, Y)=f(X)-f(Y)$, bounded by 0 . Wireframe: Graph of the response function $\mathrm{Z}=\mathrm{F}(\mathrm{X}, \mathrm{Y})$. Green and red: A triangle in the plane $\mathrm{Z}=\mathrm{X}-\mathrm{Y}$. Red: Approximate intersection of the plane and the graph of F. Purple: Approximate region in the unit square where $F(X, Y)>X-Y$ (condition 1). Blue: Approximate region in the unit square where $\mathrm{F}(\mathrm{X}, \mathrm{Y})<\mathrm{X}-\mathrm{Y}$ or $\mathrm{F}(\mathrm{X}, \mathrm{Y})=0$ (condition 2).

\subsubsection{A primitive noise-reducing AND-NOT gate}

Properties 1 and 2 do not indicate capabilities of sophisticated mathematics. An AND-

NOT response with properties 1 and 2 can be produced by mechanisms that are quite simple. Fig 3 shows that a single transistor and three resistors can be configured to accomplish this. The network output was simulated in CircuitLab, and the graph was created in MS Excel and MS 

for logic value 1, and ground stands for logic value 0 .

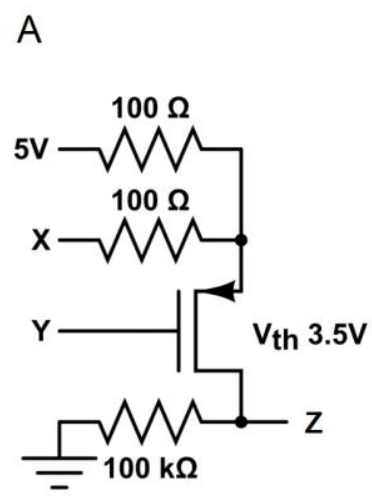

Fig 3. Single transistor AND-NOT gate that reduces noise. This minimal logic circuit

271 satisfies the noise-reducing conditions 1 and 2. A. A logic circuit consisting of one transistor and

272 three resistors. B. Engineering software simulation. Wireframe: Graph of the transistor response 273 function $\mathrm{Z}=\mathrm{F}(\mathrm{X}, \mathrm{Y})$. Green and red: A triangle in the plane $\mathrm{Z}=\mathrm{X}-\mathrm{Y}$. Red: Intersection of the 274 plane and the graph of F. Purple: Region in the unit square where $F(X, Y)>X-Y$ (condition 1). 275 Blue: Region in the unit square where $\mathrm{F}(\mathrm{X}, \mathrm{Y})<\mathrm{X}-\mathrm{Y}$ or $\mathrm{F}(\mathrm{X}, \mathrm{Y})=0$ (condition 2 ).

\subsection{Neural logic gates and flip-flops}

Fig 4 shows two logic primitives and two flip-flops. All are composed of the first type of logic primitive in Fig 4A, which can be implemented with a single neuron. 
A

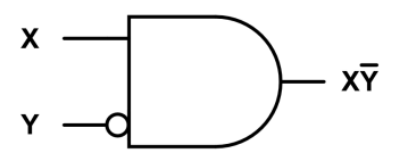

C

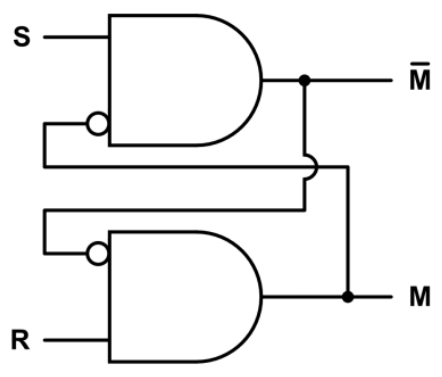

B

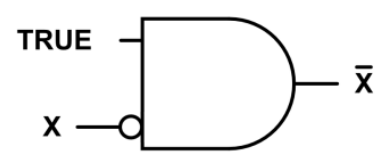

D

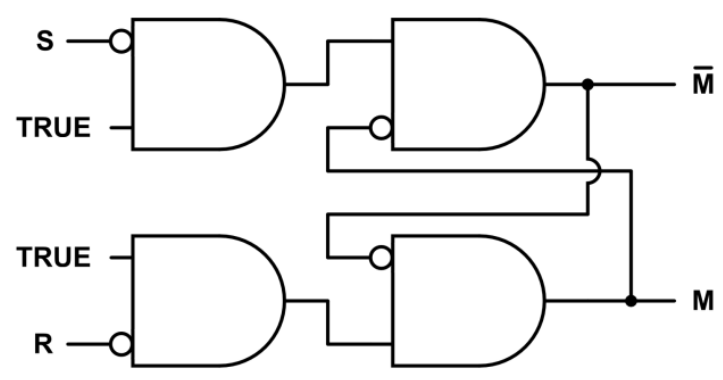

Fig 4. Neural logic gates and flip-flops. A. A symbol for an AND-NOT logic gate, with output $\mathrm{X}$ AND NOT Y. The symbol can also represent a neuron with one excitatory input $\mathrm{X}$ and one inhibitory input Y. B. An AND-NOT gate configured as a NOT gate, or inverter. C. An active low Set-Reset (SR) flip-flop. D. An active high SR flip-flop.

\subsubsection{Neural logic gates}

If $\mathrm{X}$ and $\mathrm{Y}$ are statements with truth values TRUE or FALSE, the statement "X AND NOT Y" is TRUE if and only if $\mathrm{X}$ is TRUE and $\mathrm{Y}$ is FALSE. This logic primitive is shown in Fig 4A. The figure can also represent a neuron with one excitatory input and one inhibitory input, whose response to binary inputs is X AND NOT Y by Table 1. The logic primitive NOT $X$ is TRUE if and only if $X$ is FALSE. Fig 4B shows that an AND-NOT gate with a continuously high input functions as a NOT gate.

The AND-NOT logic primitive has simplicity, efficiency, and power that have been underappreciated. It is in the minority of logic primitives that are functionally complete. (As a technicality of logic, the AND-NOT operation is not functionally complete by itself because it requires access to the input TRUE to produce the NOT operation. Only the NAND and NOR 
a high input for implementation.) Analogously to the single-neuron AND-NOT gate, the function can be implemented electronically with a single transistor and one resistor [4]. Any mechanism that can activate and inhibit like mechanisms and has access to a high activating input is a functionally complete AND-NOT gate. It may not be coincidence that the components of disparate natural signaling systems have these capabilities, e.g., immune system cells [26-29] and regulatory DNA $[30,31]$ in addition to transistors and neurons. As noted in the introduction, AND-NOT gates with analog signals can make up a powerful fuzzy logic decoder whose architecture is radically different from, and more efficient than, standard electronic decoder architectures $[2,4,5]$. Implemented with neural AND-NOT gates, these fuzzy decoders generate detailed neural correlates of the major phenomena of color vision and olfaction $[1,2]$.

\subsubsection{Neural flip-flops}

A common type of memory element used to store one bit of information in electronic computational systems is a latch or flip-flop. The more formal name is bistable multivibrator, meaning it has two stable states that can alternate repeatedly. A distinction is sometimes made between a "flip-flop" and a "latch," with the latter term reserved for asynchronous memory mechanisms that are not controlled by an oscillator. The more familiar "flip-flop" will be used here for all cases.

A flip-flop stores a discrete bit of information in an output with values usually labeled 0 and 1. This output variable is labeled $\mathrm{M}$ in Fig 4. The value of $\mathrm{M}$ is the flip-flop state or memory bit. The information is stored by means of a brief input signal that activates or inactivates the memory bit. Input $\mathrm{S}$ sets the state to $\mathrm{M}=1$, and $\mathrm{R}$ resets it to $\mathrm{M}=0$. Continual feedback maintains a stable state. A change in the state inverts the state.

Fig 4C shows an active low SR flip-flop. The $\mathrm{S}$ and $\mathrm{R}$ inputs are normally high. A brief low input $\mathrm{S}$ sets the memory bit $\mathrm{M}$ to 1 , and a brief low input $\mathrm{R}$ resets it to 0 . Adding inverters 
to the inputs produces the active high SR flip-flop of Fig 4D. The S and R inputs are normally low. A brief high input $\mathrm{S}$ sets the memory bit $\mathrm{M}$ to 1 , and a brief high input $\mathrm{R}$ resets it to 0 .

\subsubsection{Neural flip-flop simulation}

The simulation in Fig 5 demonstrates the robust operation of the NFF in Fig 4D. The simulation was done in MS Excel. The slow rise time of Set and Reset, over several delay times, is exaggerated to make the robust operation of the network clear.

Flip-flop signal strengths

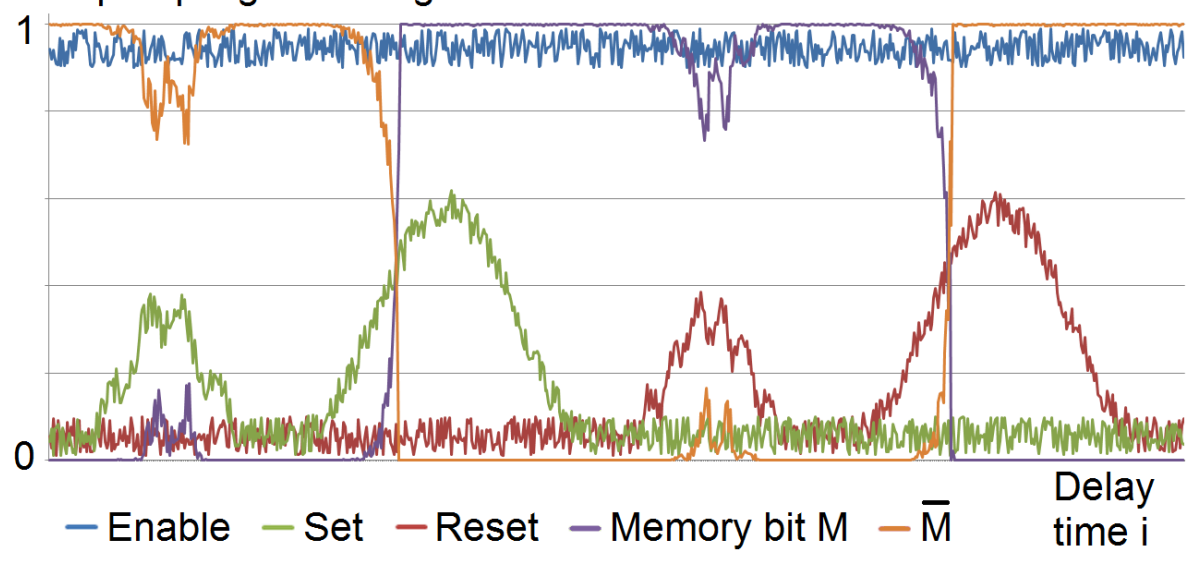

Fig 5. Simulation of an NFF operation with noise in the inputs. This simulation of the NFF in Fig 4D shows the operation is robust in the presence of moderate levels of additive noise in binary inputs. The effect of baseline noise on the memory bit is negligible, and temporary bursts of larger noise have no lasting effect.

The function $\mathrm{F}(\mathrm{X}, \mathrm{Y})$ in Fig 2 was used for the simulated neuron responses as follows. The number $t_{i}$ represents the time after $i$ neuron delay times. At time $t_{0}=0$, the outputs are initialized at $M_{0}=0$ and $\bar{M}_{0}=1$. (If both are initialized at 0 , they will oscillate until either Set or Reset is high.) At time $t_{i}$ for $i>0$, the output $Z_{i}$ of each neuron that has excitatory and inhibitory inputs $\mathrm{X}_{\mathrm{i}-1}$ and $\mathrm{Y}_{\mathrm{i}-1}$ at time $\mathrm{t}_{\mathrm{i}-1}$ is:

$$
\mathrm{Z}_{\mathrm{i}}=\mathrm{F}\left(\mathrm{X}_{\mathrm{i}-1}, \mathrm{Y}_{\mathrm{i}-1}\right)=\max \left\{0,\left[(1 / 2) \sin \left(\pi\left(\mathrm{X}_{\mathrm{i}-1}-1 / 2\right)\right)+1 / 2\right]-\left[(1 / 2) \sin \left(\pi\left(\mathrm{Y}_{\mathrm{i}-1}-1 / 2\right)\right)+1 / 2\right]\right\}
$$


Low level additive noise and baseline activity in the inputs are simulated by a computergenerated random number uniformly distributed between 0.01 and 0.1 . The noise is offset by

3410.01 so it does not obscure the high and low outputs in the graphs. The high Enabling input 342 TRUE is simulated by 1 minus noise.

Each of the medium bursts in Set and Reset is simulated by the sum of two sine functions 344 and the computer-generated noise. These signals could represent either noise bursts that are not 345 high enough to cause an error, or high input signals intended to invert the memory state but 346 sufficiently reduced by noise to cause an error. function plus noise. These signals could represent either high input signals intended to invert the memory state, substantially reduced by noise but not enough to cause an error, or noise bursts with enough amplitude to cause an error.

\subsubsection{Neural memory bank}

If information stored in short-term memory is no longer needed, active neurons consume energy without serving any useful purpose. An energy-saving function can be achieved with

354 NFFs. Fig 6 shows a memory bank of three NFFs of Fig 4D, with a fourth serving as a switch to 355 turn the memory bank on and off. The memory elements are enabled by excitatory input from 356 the switch. A large memory bank could be organized as a tree, with switches at the branch

357 points and memory elements at the leaves, so that at any time only the necessary memory 358 elements are enabled. 


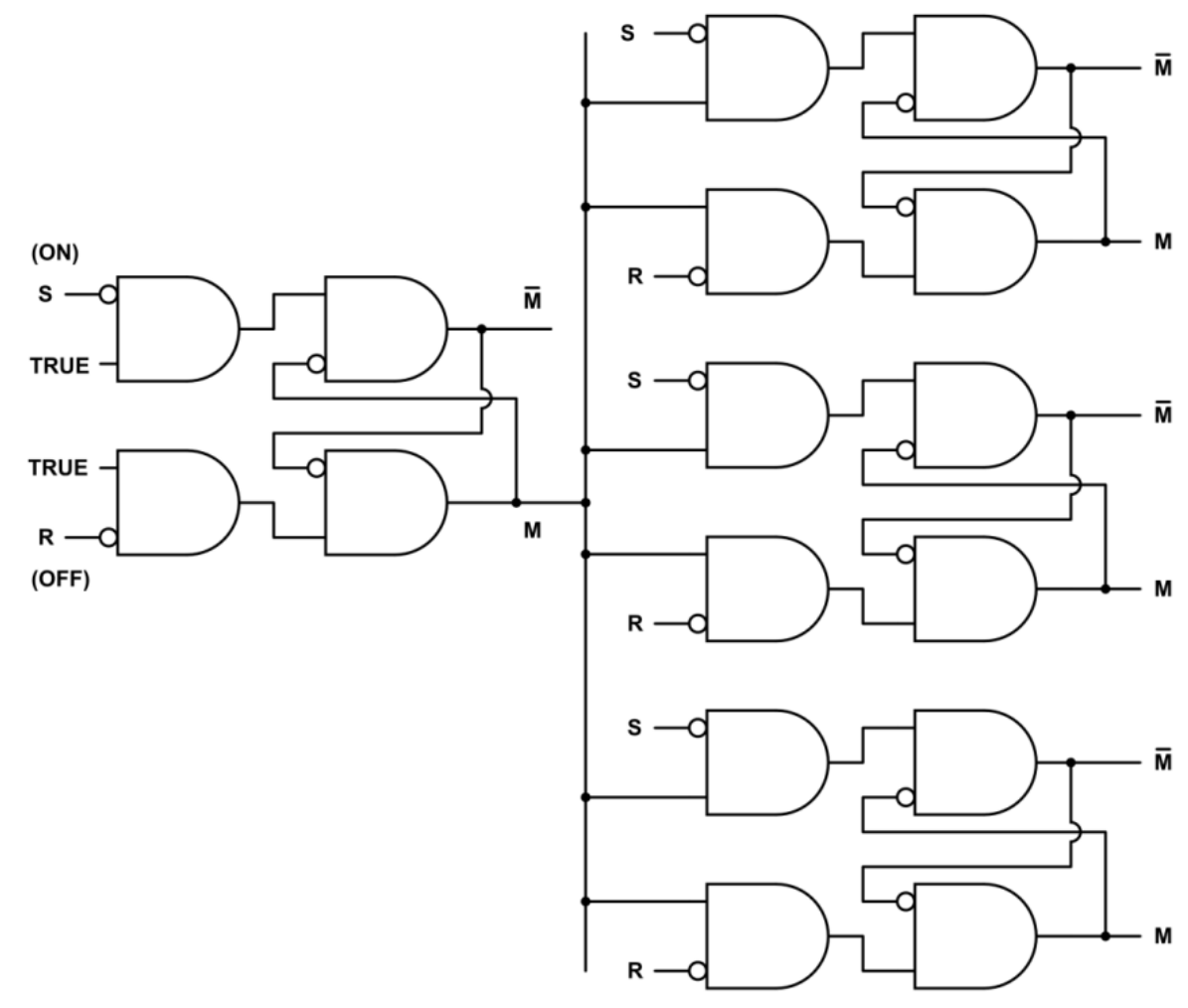

Fig 6. Neural memory bank. Three NFFs (Fig 4D) are enabled by a fourth NFF serving as an on-off switch.

\section{Results}

\subsection{Plausibility of NFFs as short-term memory mechanisms}

Flip-flops are well understood and work well as memory devices in electronic computational systems. They are capable of storing different kinds of information. It is unlikely that any short-term memory mechanism in the brain could be simpler than the NFFs in Fig 4. The simulation shown in Fig 5 illustrates that NFFs are robust in storing information. The decoders proposed in [2] can retrieve information held in NFFs. NFFs are efficient in material

370 requirements (two or four neurons), operating requirements (no physical changes besides level of 371 neuron activity), and component capability requirements (excitation and inhibition). Because

372 NFFs function dynamically, information can be stored quickly. The time required to set or reset 
an NFF is the time a signal takes to pass through two or three neurons, roughly $10-15 \mathrm{~ms}$. NFFs consume energy continuously while they are holding information. This is consistent with the brain's high energy consumption, and it may be one of the selective pressures that resulted in static mechanisms for long-term memory.

\subsection{Known memory phenomena generated by NFFs}

NFF memory banks (Fig 6) can generate the seven characteristics of neuron firing that were listed in the section on unexplained memory phenomena. For all of the characteristics, one of the two outputs of an NFF in a memory bank is identical to the sampled neuron's response. Since each NFF can store one bit of information, the number of NFFs that are required would depend on the amount of information to be recorded. To record the information conveyed by the stimulus, the visual, auditory, and sensorimotor cortexes would need to have neural structures to send the Set and Reset signals to the corresponding memory banks.

1) Before the stimulus was presented, the sampled neuron discharged at a low, baseline level. This describes one of the two NFF output neurons before the NFF state is inverted to record information. For convenience, label the output $\mathrm{M}$ before the NFF is set.

2) When the stimulus was presented, or shortly after, the neuron began to fire at a high frequency. This is the output $\mathrm{M}$ after the NFF is set by the input $\mathrm{S}$.

3) The high frequency firing continued after the stimulus was removed. This is the stored memory bit $\mathrm{M}$ after the brief NFF input $\mathrm{S}$ returns to its normal state.

4) The response was still high when the memory was demonstrated to be correct. This is the high value of $\mathrm{M}$ holding information in memory. 

bank (Fig 6) is turned off when the stored information is no longer needed, disabling all of the outputs.

6) In the trials where the subject failed the memory test, the high level firing had stopped or 7) had never begun. In instances where the high level firing had stopped, the memory bank was turned off before the memory was tested, or a distraction caused the NFF to be overwritten with new information, or noise or other errors inverted the NFF. In instances where the high

401 level firing had never begun, the NFF was not set to record the information or the NFF recorded

402 it incorrectly (for one of many possible reasons, e.g., the subject was not paying attention or was not motivated to remember). For each of these possibilities, the NFF would correctly predict

404 both the failed memory test and the corresponding observed neuron behavior.

\subsection{Testable predictions}

\subsubsection{Unknown memory phenomena generated by NFFs}

An NFF's outputs $M$ and $\bar{M}$ together predict eight unknown phenomena that could further test whether short-term memory is produced by NFFs. These predictions can be tested by

409 the same methods that were used in discovering the first seven phenomena since either $\mathrm{M}$ or $\overline{\mathrm{M}}$ 410 is predicted to be the output that produces those phenomena, and the other is predicted to be 411 nearby.

1) Along with the persistently active neuron associated with short-term memory [10, 11], another neuron has complementary output; i.e., when one is high the other is low. This is

414 predicted by $\mathrm{M}$ and $\overline{\mathrm{M}}$ in both NFFs in Fig 4 and demonstrated in the simulation of Fig 5. 
3) The two neurons are in close proximity. This is because the neurons have reciprocal inputs and are part of a small network.

4) The reciprocal inputs are inhibitory. This is shown in both NFFs in Fig 4.

5) The two neurons have some noise-reducing capability, such as responses that satisfy the inequalities 1 and 2 . Some noise-reducing capability is necessary to maintain robust binary outputs in the presence of additive noise.

6) When the neurons change states, the high state changes first. This is because the change in the neuron with the high output causes the change in the neuron with the low output. This can be seen in both NFFs in Fig 4, and it is demonstrated in the simulation of Fig 5. The change order is difficult to see in Fig 5 because of the time scale and the slow rise time of the Set and Reset inputs, but the simulation does have one neuron delay time between the completions of the two outputs' state changes.

7) The other neuron's output then changes from low to high within a few milliseconds. This happens quickly because reciprocal input from the first change causes the second within approximately one neuron delay time, regardless of how long information is held in memory. is turned off when the stored information is no longer needed, disabling all of the outputs.

\subsubsection{Predicted behavior of constructed neural networks}

Any of the networks in Fig 4 or the memory bank of Fig 6 could be constructed with neurons and tested for predicted behavior. If the single neuron in Fig 4A produces the outputs of

436 Table 1, then the predicted operations of all of the networks should follow. The NFFs are 
438

439

440

of Fig 4C is active low.) The outputs should also exhibit the properties predicted for NFFs in the preceding section.

\section{Acknowledgements}

Simulations were done with MS Excel and CircuitLab. Network diagrams were created with CircuitLab and MS Paint. Graphs were created with Converge 10.0, MS Excel, and MS Paint. The author would like to thank Arturo Tozzi, David Garmire, Paul Higashi, Anna Yoder Higashi, Sheila Yoder, and especially Ernest Greene and David Burress for their support and many helpful comments and suggestions.

\section{References}

1. Yoder L. Relative absorption model of color vision. Color Research \& Application. 2005 Aug 1;30(4):252-64.

2. Yoder L. Explicit Logic Circuits Discriminate Neural States. PloS one. 2009 Jan 7;4(1):e4154.

3. Yoder L. Explicit logic circuits predict local properties of the neocortex's physiology and anatomy. PloS one. 2010 Feb 16;5(2):e9227.

4. Yoder L, inventor. Logic circuits with and-not gate for fast fuzzy decoders. United States patent US 9,684,873. 2017 Jun 20.

5. Yoder L, inventor. Systems and methods for brain-like information processing. United States patent US 8,655,797. 2014 Feb 18.

6. McCulloch WS, Pitts W. A logical calculus of the ideas immanent in nervous activity. The bulletin of mathematical biophysics. 1943 Dec 1;5(4):115-33. 
7. Goldental A, Guberman S, Vardi R, Kanter I. A computational paradigm for dynamic logic-gates in neuronal activity. Frontiers in computational neuroscience. 2014 Apr 29;8:52.

8. Hodges A. Beyond Turing's machines. Science. 2012 Apr 13;336(6078):163-4.

9. Paninski L, Brown EN, Iyengar S, Kass RE. Statistical models of spike trains. Stochastic methods in neuroscience. 2009 Sep 24:278-303.

10. Fuster JM, Alexander GE. Neuron activity related to short-term memory. Science. 1971 Aug 13;173(3997):652-4.

11. Funahashi S, Bruce CJ, Goldman-Rakic PS. Mnemonic coding of visual space in the monkey's dorsolateral prefrontal cortex. Journal of neurophysiology. 1989 Feb $1 ; 61(2): 331-49$.

12. Kamiński J, Sullivan S, Chung JM, Ross IB, Mamelak AN, Rutishauser U. Persistently active neurons in human medial frontal and medial temporal lobe support working memory. Nature Neuroscience. 2017 Apr 1;20(4):590-601.

13. Wang XJ. Synaptic reverberation underlying mnemonic persistent activity. Trends in neurosciences. 2001 Aug 1;24(8):455-63.

14. Aksay E, Olasagasti I, Mensh BD, Baker R, Goldman MS, Tank DW. Functional dissection of circuitry in a neural integrator. Nature neuroscience. 2007 Apr;10(4):494-504.

15. Chaudhuri R, Fiete I. Computational principles of memory. Nature neuroscience. 2016 Mar;19(3):394. 
16. Cajal SR. La fine structure des centres nerveux. The Croonian Lecture. Proc. R. Soc. Lond. 1894;55:444-68.

17. Hebb DO. The organization of behavior. na; 1949.

18. Mayford M, Siegelbaum SA, Kandel ER. Synapses and memory storage. Cold Spring Harbor perspectives in biology. 2012 Jun 1;4(6):a005751.

19. Langille JJ, Brown RE. The synaptic theory of memory: a historical survey and reconciliation of recent opposition. Frontiers in systems neuroscience. 2018 Oct $26 ; 12: 52$.

20. Okun M, Lampl I. Balance of excitation and inhibition. Scholarpedia. 2009 Aug $16 ; 4(8): 7467$.

21. Kandel E, Schwartz J, Jessell T, Siegelbaum SA, Hudspeth AJ. Principles of neural science. McGraw-Hill Professional. New York, NY. 2013:160.

22. Eggermann E, Bayer L, Serafin M, Saint-Mleux B, Bernheim L, Machard D, Jones BE, Mühlethaler M. The wake-promoting hypocretin-orexin neurons are in an intrinsic state of membrane depolarization. Journal of Neuroscience. $2003 \mathrm{Mar}$ $1 ; 23(5): 1557-62$.

23. Hopfield JJ. Neurons with graded response have collective computational properties like those of two-state neurons. Proceedings of the national academy of sciences. 1984 May 1;81(10):3088-92.

24. Mysore SP, Knudsen EI. Reciprocal inhibition of inhibition: a circuit motif for flexible categorization in stimulus selection. Neuron. 2012 Jan 12;73(1):193-205. 
25. Carvalho TP, Buonomano DV. Differential effects of excitatory and inhibitory plasticity on synaptically driven neuronal input-output functions. Neuron. 2009 Mar $12 ; 61(5): 774-85$.

26. Jerne NK. The immune system. Scientific American. 1973 Jul 1;229(1):52-63.

27. Rajalingam R. Overview of the killer cell immunoglobulin-like receptor system. Immunogenetics: Methods and Applications in Clinical Practice. 2012:391-414.

28. Vilches C, Parham P. KIR: diverse, rapidly evolving receptors of innate and adaptive immunity. Annual review of immunology. 2002 Apr;20(1):217-51.

29. Uhrberg M. The KIR gene family: life in the fast lane of evolution. European journal of immunology. 2005 Jan 1;35(1):10-5.

30. Robinson R. Mutations change the boolean logic of gene regulation. PLoS biology. 2006 Mar 28;4(4):e64.

31. Stepanova M, Tiazhelova T, Skoblov M, Baranova A. A comparative analysis of relative occurrence of transcription factor binding sites in vertebrate genomes and gene promoter areas. Bioinformatics. 2005 Feb 4;21(9):1789-96. 